\title{
Apport du radar Géologique à la connaissance de la répartition spatiale de dépôts de tempêtes des lagunes de Sidi Moussa et Oualidia (côte ouest atlantique marocaine)
}

\author{
Khalid MEHDI ${ }^{1}$, Franck LAVIGNE ${ }^{2}$, Khalid EL KHALIDI ${ }^{1}$, Samira MELAS ${ }^{1}$, \\ Clérmont VIRMOUX ${ }^{2}$, Bendahhou ZOURARAH ${ }^{1}$, \\ Daniel BRUNSTEIN ${ }^{2}$, Alexandre SAHAL ${ }^{2}$
}

1. Université Chouaïb Doukkali, Faculté des Sciences El Jadida, Laboratoire Géosciences Marines et Sciences du Sol -URAC 45, Maroc. mehdkhalid@gmail.com

2. Université Paris 1 Panthéon-Sorbonne, Laboratoire de Géographie Physique UMR 8591 CNRS, France.

\section{Résumé :}

Les régions littorales constituent des milieux fragiles et sous la dépendance de risques naturels nombreux parmi lesquels, les tempêtes, les cyclones, les tsunamis et la remontée actuelle et future du niveau général des mers.

Cependant seuls les indices de tempêtes historiques sur les littoraux sont nombreux et permettent de mettre en évidence les actions conjuguées de la houle, de la marée et de vents violents sur les littoraux sableux. Parfois ces indices peuvent également correspondre, dans certaines régions, à des témoignages de la mise en place de dépôts sous l'effet de vagues gigantesques produites par des ébranlements sismiques sous marins. Des investigations récentes réalisées par l'équipe du projet ANR Maremoti, et menées au niveau du littoral des Doukkala ont permis la mise en évidence à proximité de la lagune de Oualidia et de Sidi Moussa, de l'existence d'un éventail sableux correspondant à une morphologie comparable à un delta intra lagunaire progradant sur la lagune par l'action conjuguée des vagues et de vents violents et formant un éventail de débordement. Ce dépôt est constitué de sable remobilisé sur le rivage et déversé vers la lagune par-dessus le cordon littoral, durant une tempête (ou un tsunami). Ces dépôts sont parfaitement visibles sur la photographie aérienne de la région (figure 1). L'analyse des accumulations de part et d'autre de cette lagune a également mis en évidence l'existence de dépôt de galets de tailles variées, plaqués aux niveaux des falaises vives du cordon. Ces galets carbonatés qui ne peuvent provenir d'aucune formationgéologique continentale et/ou littorale pourraient être issus de l'érosion des affleurements rocheux jurassico-crétacés de la plate-forme adjacente (CIRAC et al., 1979) et auraient été mis en place lors de tempêtes exceptionnelles. Une étude géophysique par radar géologique des tendances spatiales et de l'architecture sédimentaire de deux dépôts alluviaux a été réalisée, le long de la côte ouest atlantique marocaine au niveau des lagunes de Sidi Moussa et d'Oualidia (figure 1). 


\section{Introduction}

Les littoraux sableux sont fréquemment soumis à des processus hydrodynamiques particulièrement puissants. Parmi ceux-ci, les tsunamis (rares mais souvent destructeurs) liés en général à des phénomènes sismiques de magnitude importante et dont l'épicentre se situe en milieu marin, constituent des évènements majeurs en termes d'évolution rapide des environnements littoraux. Curieusement, alors que de nombreux témoignages historiques font référence au raz de marée associé au tremblement de terre de Lisbonne (une vague de 8 à $10 \mathrm{~m}$ de hauteur aux environs des remparts de la ville d'El Jadida) on n’observe pas de témoins morphologiques et sédimentologiques comparables à ceux décrits dans le Golfe de Cadix (LUQUE et al., 2001).

Le littoral atlantique marocain montre sur de très grandes distances soit des littoraux sableux actuels et subactuels peu ou pas consolidés (par exemple entre Larache et Tanger), soit des plages aux matériaux quaternaires dunaires consolidés (calcarénites) constituant des falaises (environs de Rabat) ou bien encore des lapiez côtiers très découpés et déchiquetés (Sud de Rabat, environs de Casablanca).

La région des Doukkala, entre El Jadida au Nord et Safi au Sud (figure 1), présente pour sa part, un littoral assez inhospitalier avec une succession de falaises gréseuses très entaillées par les houles et séparant de petites criques constituées par des dépôts sableux. C'est au niveau de la lagune d'Oualidia et de Sidi Moussa (figure 1) que nous avons observé un important débordement sableux conséquence d’une forte tempête (washover). L'existence de ce dépôt de tempête est l'indice d'un évènement hydrodynamique très important que nous ne pouvons mettre en relation que soit avec une tempête exceptionnelle soit avec l'impact d'une vague générée par un séisme sous marin de haute magnitude et engendrant un raz de marée.

L'objectif de cette étude est de mettre en évidence par la méthode du radar géologique les caractéristiques sédimentologiques et morphométriques de ces dépôts de tempête ainsi que leur répartition spatiale, pour expliquer les mécanismes de mise en place de ces dépôts et leur relation avec une tempête exceptionnelle ou un tsunami.

\section{Matériel et méthodes}

Le GPR (Ground Penatrating Radar) ou radar géologique fonctionne sur la base de la propagation de la réflexion et de la diffraction d'ondes électromagnétiques dans un milieu. Il émet un champ électromagnétique et va enregistrer les différentes réponses que va renvoyer le milieu prospecté. Ces réponses vont rendre compte des différences diélectriques du sol. L’image du sous sol qui est obtenue est le radargramme, qui constitue une image pouvant ensuite subir différents traitements afin d'améliorer sa lecture. Dans les deux milieux lagunaires prospectés plusieurs profils ont été mesurés (figure 2) par un radar équipé d’une antenne émettrice dont la fréquence était de $500 \mathrm{MHz}$. Afin de compléter les mesures radar, des prélèvements par tarière, des tranchées, ainsi que des données de photographies aériennes ont été utilisés. 


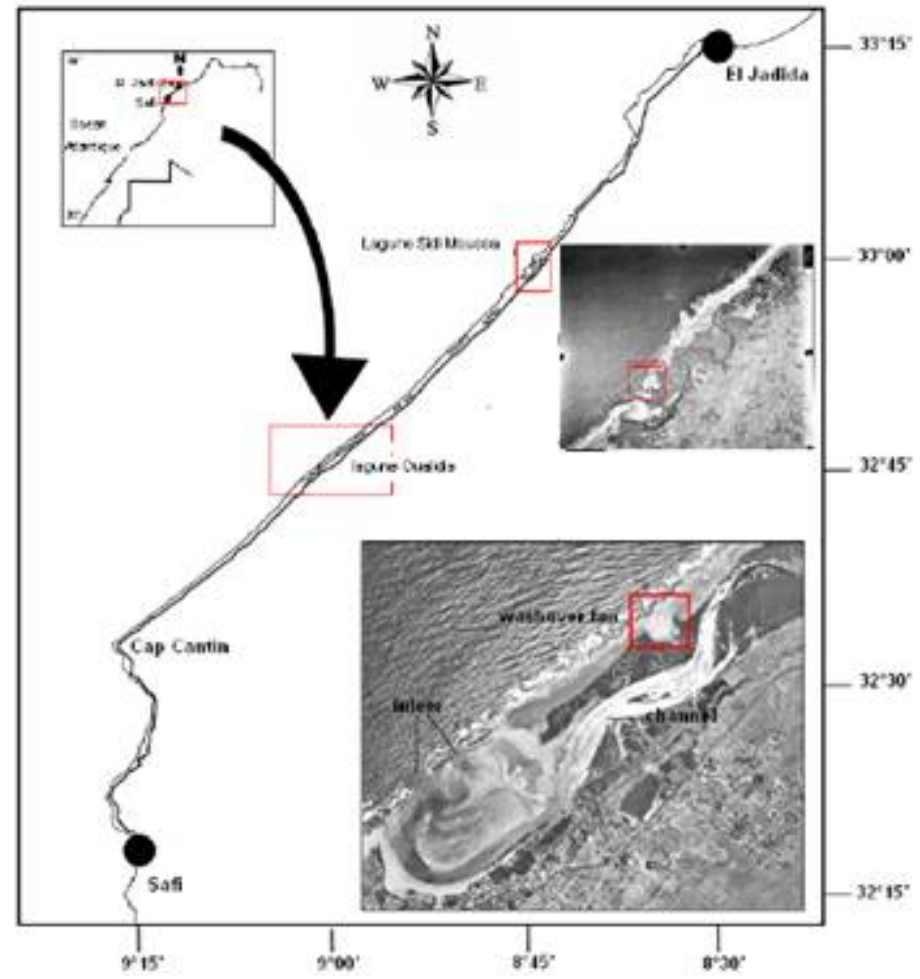

Figure 1. Situation géographique de la région étudiée.
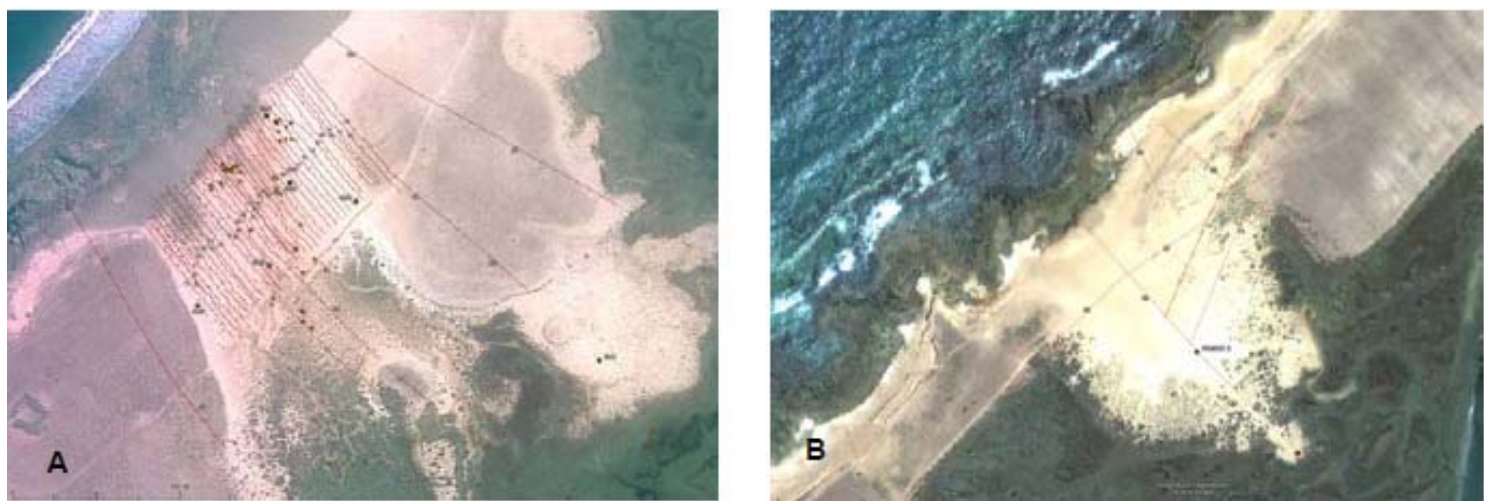

Figure 2. Profils radar réalisés sur les dépôts de la lagune de Sidi Moussa (A) et de la lagune de Oualidia (B).

\section{Résultats}

Les images radar analysées au niveau des dépôts de la lagune de Sidi Moussa, montrent des dizaines de rochers de taille plurimétrique à une centaine mètres de la côte. Les profils du radar géologique (figure 3), révèlent quelques rochers enterrés, qui sont progressivement exhumés par les tempêtes. Par conséquent, ces dépôts de washover ont été mis en place, vraisemblablement, par plusieurs séquences individuelles impliquant un tsunami, qui est le seul événement capable de déplacer des rochers de taille plurimétrique dans ce type de dépôt. 
Dans la lagune de Oualidia, les images radar analysées montrent parfois des dépôts de tempête stratifiés à limites parallèles à subparallèles avec des marqueurs peu inclinés (figure 4) ; dans d'autres cas on constate des réflecteurs avec des formes sigmoïdes et obliques. La forme géométrique des réflecteurs des images radar de Oualidia laisse supposer que les dépôts ce sont faits dans des eaux peu profondes, stagnantes, pouvant correspondre à des dépôts de delta.

Ce résultat est cohérent avec les données des photos aériennes, qui montrent une progradation de ces dépôts depuis les années 1950, conséquence d’une série de tempêtes ayant marqué la région du sahel de Doukkala.

\section{Conclusion}

Les résultats obtenus par les campagnes de prospection radar sur les deux lagunes de Sidi Moussa et Oualidia, nous ont permis de mettre en évidence la présence de dépôts de tempêtes d'origines tsunamiques, en relation le tsunami de 1755 qui a frappé la ville de Lisbonne et la côte atlantique marocaine. Les données de terrain en cours de traitement (carottes et datations) permettront de valider ou non cette hypothèse.

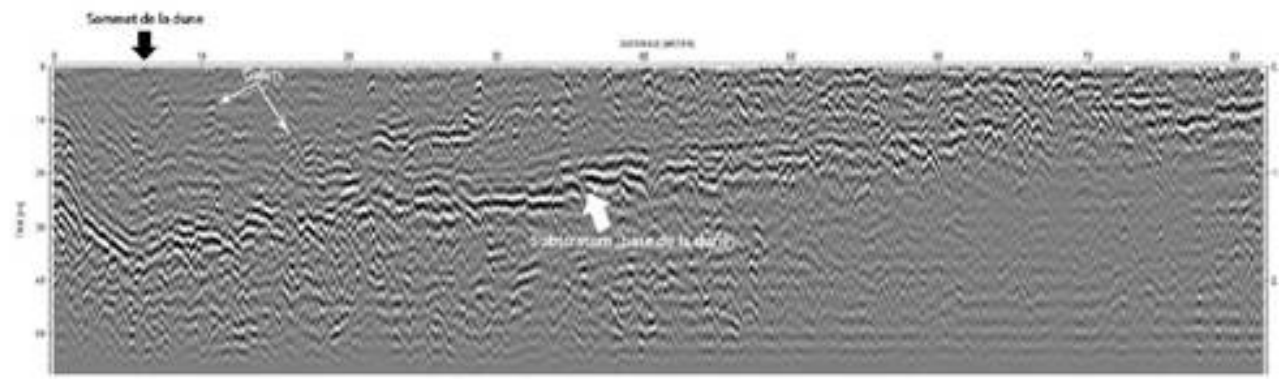

Figure 3. Profil radar au niveau du washover de Sidi Moussa.

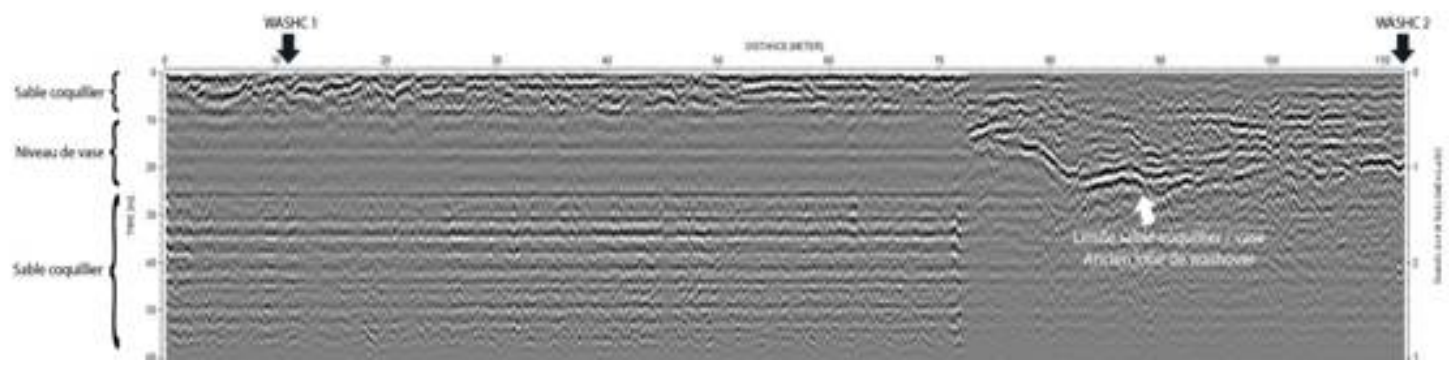

Figure 4. Profil radar le long du washover de Oualidia.

\section{Références bibliographiques}

CIRAC P., FAUGERES J.C et GAYET J. (1979). Résultats préliminaires d'une reconnaissance sédimentaire du plateau atlantique marocain. Bull. Inst. Géol. Bassin d'Aquitaine, Bordeaux, 25, pp 69- 81.

LUQUE L., LARIO J., ZAZO C., GOY J.L., DABRIO .C.J., SILVA P.G. (2001). Tsunami deposits as palaeosismic indicators: examples from the Spanish coast. Acta Geologica Hispanica 3-4, pp 197-211. 